\title{
Effects of Natural Products on Nonalcoholic Fatty Liver Disease through Nrf2 Epigenetic Regulation
}

\author{
Jiayue $\mathrm{Cao}^{1, *}$ \\ ${ }^{1}$ School of Life Science, 999077 Sha Tin, Hong Kong, China
}

\begin{abstract}
Non-alcoholic fatty liver disease has become one of the common illnesses among urban dwellers worldwide. Many consider natural products as better options for medicine and diet supplements. In recent years, researchers found that natural products can prevent human diseases by reactivating silencing genes through epigenetic regulation. They also discovered nuclear factor erythroid-2-related factor-2 (Nrf2) as is an important factor in the response of anti-oxidation has a close relation to lipogenesis. Studies showed with the higher expression of DNA methyl-transferases, the higher methylation of Nrf2 promoter occurred. With the high methylation rate of the Nrf2 promoter, the expression of Nrf2 reduced thus enhanced lipid accumulation, which may cause NAFLD. Furthermore, with lower expression of Nrf2, the downstream antioxidant genes' expression decreased, which may cause oxidative stress to human body cells. Understanding the pathway of Nrf2 and NAFLD can shed a light on discovering new development of drugs. This paper reviews several natural products and their mechanisms of alleviating NAFLD and hopes to bring in new perspectives on the treatment and the development of new drugs on NAFLD.
\end{abstract}

\section{Introduction}

Non-alcoholic fatty liver disease (NAFLD) is a frequent cause of chronic liver diseases due to the prevalence of high-fat diet and obesity in modern society. It refers to a broad spectrum of liver pathology which varies from simple steatosis to non-alcoholic steatohepatitis (NASH) and even cirrhosis[1-3].

Nuclear factor erythroid-2-related factor-2 (Nrf2) is an crucial transcription factor in the response of cells to oxidative stress and a central key for cells to resist antioxidant damage, which control the composition of oxidative response element (ARE) dependent genes to direct the physiological reaction of free radical and antioxidant imbalance[4]. It exists in organs like liver and kidney and a variety of tissues and cells. Under normal physiological conditions, Nrf2 can bind to KEAP1. The dissociation of KEAP1 and Nrf2 is a result of response to oxidative and electrophilic stress because it phosphorylates and induces conformational changes of Nrf2. The dissociated Nrf2 is transferred to the nucleus, where it dimerizes with MAF family proteins and activates the ARE pathway, thereby improving transcription of various cell-protective genes, including HO-1, NQO1, SOD, GCLM, and MRP1. The antioxidative stress defense mechanism of the Nrf2-ARE pathway is a potential cancer prevention and treatment[5-7]. Recent research revealed that Nrf2 is related to NAFLD through multiple pathways[8].

Growing evidence indicates that epigenetic modifications control gene expression, which involves DNA methylation and histone modification. DNA methyl-transferases (DNMTs) catalyze the methylation of $\mathrm{CpG}$ island cytosine in DNA, interfering with the binding of RNA polymerase and DNA to reduce transcription. Major DNMTs, including DNMTs 1, 3A, and 3B, transfer methyl groups from $\mathrm{S}$ adenosylmethionine (SAM) to cytosine[9].

In recent studies, it is discovered that natural products have shown promises of preventing human diseases through epigenetic regulation by reactivating silencing genes. This paper reviews several natural products and their mechanisms of relieving NAFLD and hopes to provide new ideas on the treatment and the development of new drugs on NAFLD.

\section{Mechanism of Nrf2 signaling pathway action in NAFLD}

Under the basal condition, Keap1 protein promotes the proteasomal degradation of Nrf2 as its inhibitor, but it allows Nrf2 to stabilize and translocate into the nucleus to regulate transcription numerous genes under oxidative stress, which are vital in protection against oxidative stress[10-11](Fig. 1).

Nrf2 may also be related to the reduction in triglyceride level and decrease in the expression of lipogenesis-related genes. Nrf2 can protect cells from steatosis by inhibiting lipogenesis and promoting fatty acid oxidation because ofthe activation of AREcontaining transcription factors that adjust adipocyte differentiation and adipogenesis and by the protection against redox-dependent inactivation of metabolic

\footnotetext{
*Corresponding author: caojiayue0823@163.com
} 
enzymes[12]. Apart from enhancing the expression of antioxidant enzymes such as HO-1, Nrf2 can also participate in the anti-oxidation processes in the liver[13], which can reduce lipid growth by controlling lipogenesis.

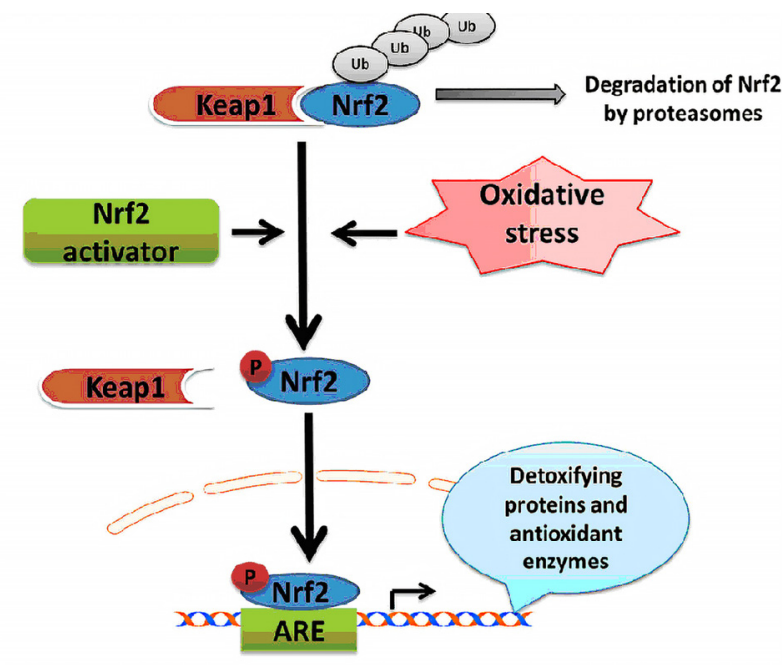

Fig. 1. Nrf2 signaling pathway

\section{Effects of natural products on NAFLD}

DNA methylation is one of the critical epigenetic mechanisms for controlling gene expression in eukaryotes. Based on several studies, the Nrf2 promoter is known to be highly methylation in NAFLD cells[1719], however, the underlying mechanism is still not completely explored.

Resveratrol is a natural polyphenol and phytoalexin, which has abundant biological properties as antiinflammatory, antioxidant, anti-tumor, anti-aging, cardiovascular protective and chemo-protective, antidiabetes, etc[14-16]. Zhao et al. suggested that DNA demethylation may create a positive link for reactivating the Nrf2 signaling in Alzheimer's disease cellular model[20].

According to Hosseini et al., resveratrol could reduce the DNA methylation rate of the Nrf2 promoter in mice fed by a high-fat diet (Fig. 2)[21]. After feeding HFD, the level of Nrf2 methylation in those mice was significantly high as well as mRNA and the protein expression level of Nrf2. By adding resveratrol, the level of Nrf2 mRNA and the expression of Nrf2 protein increased, together with the expression of downstream antioxidant, sterol regulatory element-binding transcription factor 1c (SREBP-1c), and fatty acid synthase (FAS) genes.

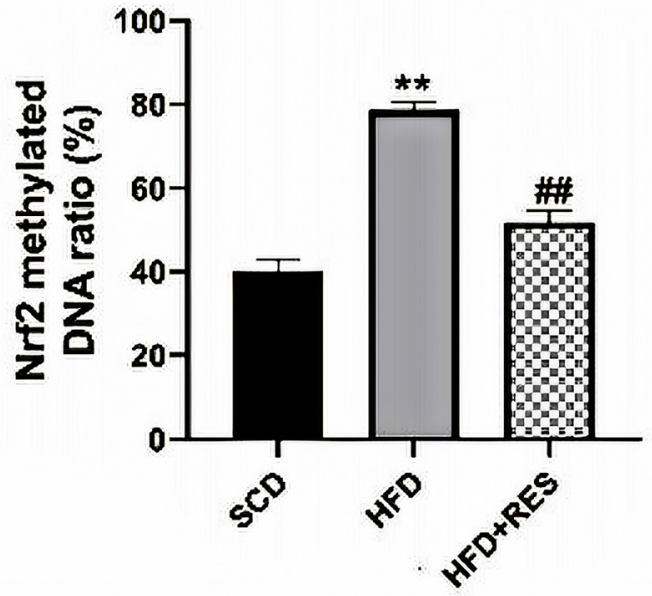

Fig. 2. Nrf2 methylated DNA ratio[21]

Aged citrus peel (Chenpi) is made from the dried peel of orange or tangerine belonging to the family Rutaceae[21]. Mainly composed of essential oils, pectin, phenolic acid, flavonoids, and polymethoxyflavonoids (PMFs), the citrus peel can play an effective role in liver health. condition According to Lin et al., Chenpi triggered the antioxidative pathway induced by $\mathrm{Nrf} 2$. Besides, Chenpi can limit the expression of DNA methylation transferase(DNMT) 1 and $3 \mathrm{~A}$, which are the main enzymes in transferring methyl-CpG sites(Fig. 3), to decrease the methylation level of the Nrf2 promoter. Taken together, Chenpi silences DMNT and decreases the rate of Nrf2 gene promoter methylation[23], resulting in demethylating the Nrf2 promoter.

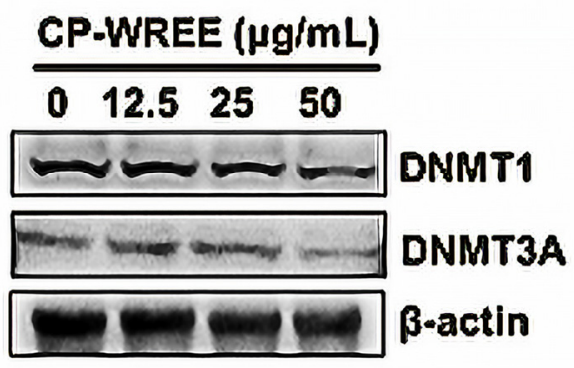

Fig. 3. Expression of DNMT1 and DNMT3A[23]

Sulforaphane, produced from cruciferous vegetable plants, has been proved to inhibit or retard tumor incidence and progression in models of breast, colon, stomach, and lung cancer[24]. According to Zhou et al., sulforaphane can create homeostatic protection of cells and tissues against carcinogens[25], by repressing DNMT1 protein expression and lessening DNMT1 activity to lead to the demethylation of the promoter region of Nrf2 and increased activation of Nrf2, inducing the transcription of the defensive enzymes glucuronyl transferase(UGTs).

Anthocyanidins, abundant in many fleshy fruits such as berries, red grapes, etc. are important healthpromoting phytochemicals[27]. Anthocyanidins give the intense color to many fruits and vegetables. Among all types of anthocyanidins, delphinidin is reported to be 
one of the most efficient compounds. Delphinidin has been reported to specifically inhibit inflammatory signals[28]. According to Kuo et al., delphinidin could reduce the rate of $\mathrm{CpG}$ methylation in the $\mathrm{Nrf} 2$ promoter region. And decreased the expression of DNMT1 and DNMT3A(Fig. 4)[29]. The decreased methylated ratio of $\mathrm{Nrf} 2$ gene promoter causes the reactivate of $\mathrm{Nrf2}$ signaling pathway which may decrease the risk of NAFLD.

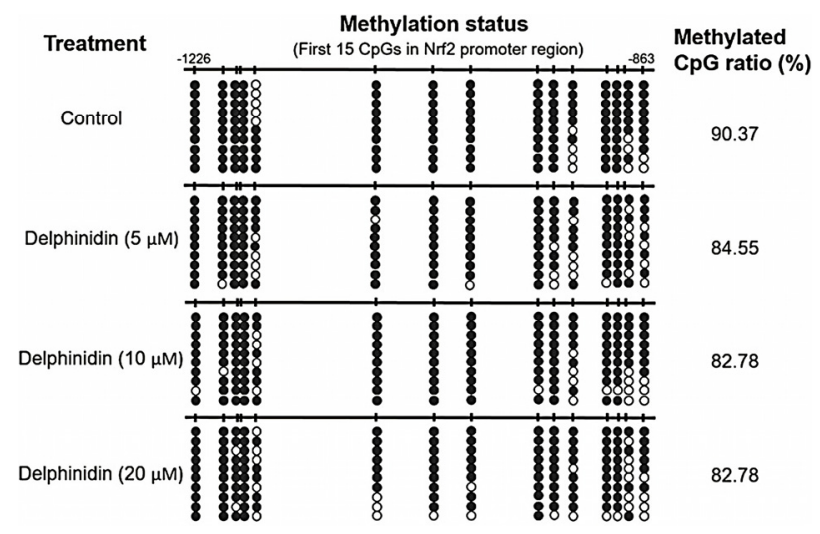

Fig. 4. Methylated CpG ratio[29].

\section{Conclusion}

This paper presents an overview of the up-to-date knowledge regarding the epigenetics of Nrf2 gene promoter of NAFLD. The main mechanism of these natural products is to silence the expression of DMNTs which can lower the methylation rate of $\mathrm{Nrf} 2$ and lead to the enhancement of Nrf2 and downstream antioxidant enzymes gene expression. These natural productsprovide new options on the treatment of NAFLD (Fig. 5).

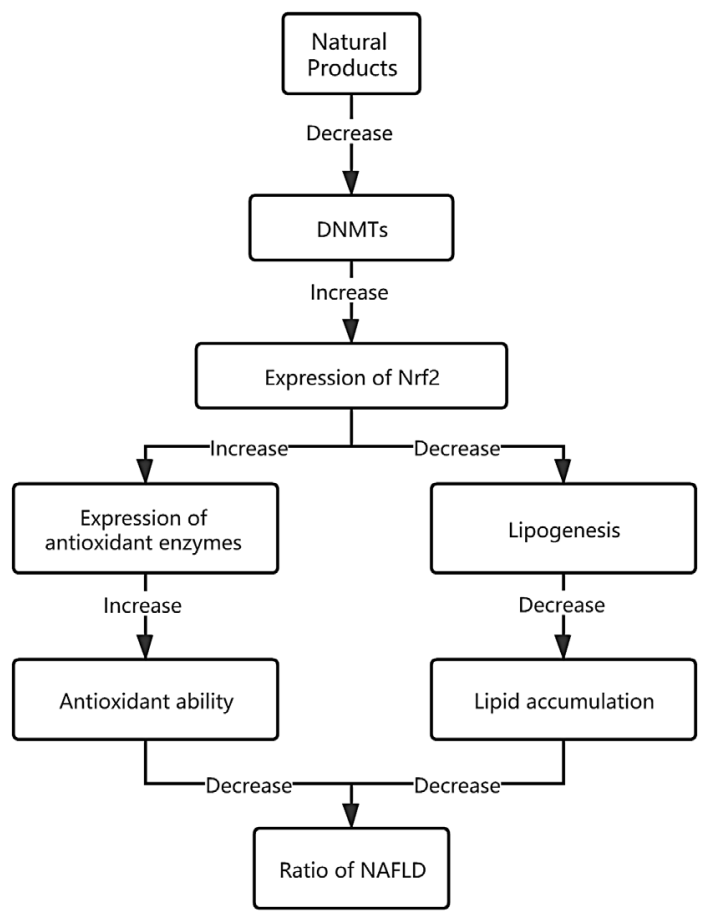

Fig. 5. Natural products decrease ratio of NAFLD

\section{Prospect}

The main clinical treatment for NAFLD is surgery. However, most patients concern the risk of surgical treatment. Patients now have more options, like specific drugs to balance lipid metabolism for NAFLD treatments, instead of the traditional operation. The active compound from the natural products has gathered more and more attention for the past few years. As for the invention of drugs on NAFLD, mainly consists of phenolic acids, alkaloids, flavonoids, etc. These compounds have different chemical structures but with similar effects and results. Hence, the epigenetic mechanism plays a vital role in the diagnosis and treatment of NAFLD. So by understanding the basic effect of these natural products, it can provide more clues on solving the underlying pathway of NAFLD. Fatty liver disease is known to relate to oxidation. Nrf2 served as the major antioxidant signaling pathway, so by learning the pathway of Nrf2 with NAFLD, we hope to discover more suitable drug supplement or dietary plan for NAFLD patients or susceptible population.

\section{References}

1. Milić S, Lulić D, Štimac D. Non-alcoholic fatty liver disease and obesity: biochemical, metabolic and clinical presentations. World journal of gastroenterology: WJG. 20(28):9330 (2014).

2. Dietrich P, Hellerbrand C. Non-alcoholic fatty liver disease, obesity and the metabolic syndrome. Best practice \& research Clinical gastroenterology. 28(4):637-53 (2014).

3. Wree A, Broderick L, Canbay A, Hoffman HM, Feldstein AE. From NAFLD to NASH to cirrhosisnew insights into disease mechanisms. Nat Rev Gastroenterol Hepatol; 10:627-636 (2013)

4. Huang, Y., W. Li, Z.Y. Su and A.N. Kong. The complexity of the Nrf2 pathway: beyond the antioxidant response. J. Nutr. Biochem. 26: 14011413 (2015).

5. Deshmukh P, Unni S, Krishnappa G, Padmanabhan B. The Keap1-Nrf2 pathway: promising therapeutic target to counteract ROS-mediated damage in cancers and neurodegenerative diseases. Biophys Rev. 9(1):41-56 (2017).

6. Jaramillo MC, Zhang DD. The emerging role of the Nrf2-Keap1 signaling pathway in cancer. Genes Dev. 27(20):2179-91 (2013).

7. Kuo, Hsiao-Chen Dina; Wu, Renyi; Li, Shanyi; Yang, Anne Yuqing; Kong, Ah-Ng. Anthocyanin Delphinidin Prevents Neoplastic Transformation of Mouse Skin JB6 P+ Cells: Epigenetic Re-activation of Nrf2-ARE Pathway. The AAPS Journal, 21(5) (2019).

8. Zhang, X., Asllanaj, E., Amiri, M., PortillaFernandez, E., Bramer, W. M., Nano, J., Ghanbari, M. Deciphering the role of epigenetic modifications 
in fatty liver disease: A systematic review. European Journal of Clinical Investigation (2020).

9. Lee, J.H., T.O. Khor, L. Shu, Z.Y. Su, F. Fuentes and A.N. Kong. Dietary phytochemicals and cancer prevention: Nrf2 signaling, epigenetics, and cell death mechanisms in blocking cancer initiation and progression. Pulm. Pharmacol. Ther. 137: 153-171, (2013).

10. Guo Y, Yu S, Zhang C, Kong A-NT. Epigenetic regulation of Keap1-Nrf2 signaling. Free Radical Biology and Medicine. 88:337-49 (2019).

11. Cheng D, Wu R, Guo Y, Kong A-NT. Regulation of Keap1-Nrf2 signaling: the role of epigenetics. Current opinion in toxicology. 1:134-8 (2016).

12. Shen, Bingyu; Zhao, Chenxu; Wang, Yue; Peng, Yi; Cheng, Jiaqi; Li, Zheng; Wu, Lin; Jin, Meiyu; Feng, Haihua . Aucubin inhibited lipid accumulation and oxidative stress via Nrf2/HO-1 and AMPK signalling pathways. Journal of Cellular and Molecular Medicine. (2019).

13. S. Tao, R. Justiniano, D.D. Zhang, G.T. Wondrak, The Nrf2-inducers tanshinone I and dihydrotanshinone protect human skin cells and reconstructed human skin against solar simulated UV, Redox Biol. 1 532-541 (2013).

14. Nakao, S.; Mabuchi, M.; Wang, S.; Kogure, Y.; Shimizu, T.; Noguchi, K.; Tanaka, A.; Dai, Y. Synthesis of resveratrol derivatives as new analgesic drugs through desensitization of the TRPA1 receptor. Bioorg. Med. Chem. Lett. 27(14), 3167-3172 (2017).

15. Kolahdouz Mohammadi, R.; Arablou, $\mathrm{T}$. Resveratrol and endometriosis: In vitro and animal studies and underlying mechanisms (Review). Biomed. Pharmacother. 91 220-228 (2017).

16. Aldawsari, F.S.; Aguiar, R.P.; Wiirzler, L.A.M.; Aguayo-Ortiz, R.; Aljuhani, N.; Cuman, R.K.N.; Medina-Franco, J.L.; Siraki, A.G.; VelázquezMartínez, C.A. Anti-inflammatory and antioxidant properties of a novel resveratrol-salicylate hybrid analog. Bioorg. Med. Chem. Lett. 26(5), 1411-1415 (2016).

17. Zhang, X., Asllanaj, E., Amiri, M., PortillaFernandez, E., Bramer, W. M., Nano, J. Ghanbari, M.. Deciphering the role of epigenetic modifications in fatty liver disease: A systematic review. European Journal of Clinical Investigation (2020).

18. Pirola CJ, Gianotti TF, Burgueno AL, Rey-Funes M, Loidl CF, Mallardi P et al. Epigenetic modification of liver mitochondrial DNA is associated with histological severity of nonalcoholic fatty liver disease. Gut 62:1356-63 (2013).

19. Vanessa D. de Melloa, Ashok Mattea, Alexander Perfilyev, Ville Männistö, Tina Rönn, Emma Nilssonb et al. Human liver epigenetic alterations in nonalcoholic steatohepatitis are related to insulin action. Epigenetics 12:287-95 (2017).

20. Zhao F, Zhang J, Chang N. Epigenetic modification of Nrf2 by sulforaphane increases the antioxidative and anti-inflammatory capacity in a cellular model of Alzheimer's disease. European journal of pharmacology. 824 1-10 (2018).

21. Hosseini H, Teimouri M, Shabani M, et al. Resveratrol alleviates non-alcoholic fatty liver disease through epigenetic modification of the Nrf2 signaling pathway[J]. The international journal of biochemistry \& cell biology, 119:105667 (2019).

22. Gualdani, R., M.M. Cavalluzzi, G. Lentini and S. Habtemariam. The chemistry and pharmacology of Citrus limonoids. Molecules 21: 1530-1568 (2016).

23. Lin, Zi-Han; Chan, Yen-Fan; Pan, Min-Hsiung; Tung, Yen-Chen; Su, Zheng-Yuan. Aged Citrus Peel (Chenpi) Prevents Acetaminophen-Induced Hepatotoxicity by Epigenetically Regulating Nrf2 Pathway. The American Journal of Chinese Medicine, 47(8), 1833-1851 (2019).

24. Chikara S, Nagaprashantha LD, Singhal J, Horne D, Awasthi S and SinghalSS: Oxidative stress and dietary phytochemicals: Role in cancer chemoprevention and treatment. Cancer Lett 413: 122-134, (2018).

25. Zhou, Jia-Wei; Wang, Min; Sun, Nuan-Xin; Qing, Ying; Yin, Teng-Fei; Li, Cui; Wu, Dong. Sulforaphane-induced epigenetic regulation of Nrf2 expression by DNA methyltransferase in human Caco-2 cells. Oncology Letters, 18:2639-2647 (2019).

26. Barrière, G.; Tartary, M.; Rigaud, M. Metformin: A rising star to fight the epithelial mesenchymal transition in oncology. Anticancer Agents Med. Chem. 13 333-340 (2013).

27. Hou, De-Xing; Fujii, Makoto; Terahara, Norihiko; Yoshimoto, Makoto. Molecular Mechanisms Behind the Chemopreventive Effects of Anthocyanidins. Journal of Biomedicine and Biotechnology, 2004(5), 321-325 (2004).

28. Seong AR, Yoo JY, Choi K, Lee MH, Lee YH, Lee $\mathrm{J}$, et al. Delphinidin, a specific inhibitor of histone acetyltransferase, suppresses inflammatory signaling via prevention of NF- $\kappa \mathrm{B}$ acetylation in fibroblastlike synoviocyte MH7A cells. Biochem Biophys Res Commun.. 410(3):581-6 2011.

29. Kuo, Hsiao-Chen Dina; Wu, Renyi; Li, Shanyi; Yang, Anne Yuqing; Kong, Ah-Ng. Anthocyanin Delphinidin Prevents Neoplastic Transformation of Mouse Skin JB6 P+ Cells: Epigenetic Re-activation of Nrf2-ARE Pathway. The AAPS Journal, 21(5), 21-83 (2019). 